\title{
INORGANIC ORGANIC COMPOSITE MATERIALS AS ABSORBERS FOR ORGANIC SOLVENTS
}

\author{
V. Gerhard, H. Schmidt and U. Dreier \\ Composite Department, Institut für Neue Materialien GmbH, Im Stadtwald, Geb. 43, \\ D-66123 Saarbrücken, Germany
}

\begin{abstract}
Inorganic organic composite materials have been developed in the form of non-porous films on various substrates and as porous materials with specific surface areas of about $700 \mathrm{~m}^{2} / \mathrm{g}$. Both types of materials have been tested for absorption and adsorption of solvents from the gas phase (butyric acetate, benzene, toluene and xylene). The results show that the non-porous films could be loaded with solvents up to $30 \mathrm{wt} \%$ and unloaded at $130^{\circ} \mathrm{C}$ within a few minutes. The porous materials could be loaded up to $38 \mathrm{wt} \%$. The kinetics of desorption are slowed down to values of some hours, being however still fast compared to activated carbon. Adsorption in wet atmospheres do not affect adsorption capacity but leads to a decrease of adsorption kinetics due to a slowing down of the diffusion or a replacement of $\mathrm{H}_{2} \mathrm{O}$ against solvent within the pores.
\end{abstract}

\section{INTRODUCTION}

Porous materials are used as adsorbers and catalysing materials, for scientific examination and technical applications. Common adsorption materials are activated carbon, activated aluminum oxide, silica gel or zeolithes. These materials normally show high adsorption capacities but also very long desorption times (e. g. activated carbon: $>1$ day). The active surface of adsorbers can be poisoned by compounds, which can not be desorbed any more. In contrast, diffusion controlled absorption processes do not show these disadvantages. Since the material properties of inorganic organic hybrid materials can be tailored in wide ranges by variation of their composition and the process parameters during their synthesis, these composites are predestined as absorber materials. Composites, which are adapted to special separation problems can be applied as coatings on different surfaces by common coating techniques, e. g. dip or spray coating, or can be used as bulk materials for absorption columns [1,2].

The aim of the investigations was to compare the sorption behaviour of inorganic organic composites and commonly used absorbers for different organic solvents.

\section{EXPERIMENTAL}

The inorganic organic composite coating materials were prepared by adding dropwise the stoichiometric amount of $0.1 \mathrm{n}$ hydrochloric acid to the organically modified alkoxysilane or mixture of different silanes. Two phases are perceptible. The mixture is stirred at room temperature for $3 \mathrm{~h}$, until the phase separation has disappeared. The material is applied by dip or spray coating and is then thermally cured at $130^{\circ} \mathrm{C}$. Inorganic organic composites were prepared as bulk materials from solutions of tetramethylorthosilicate and methyltriethoxysilane by adding dropwise the stoichiometric amount of $6 \mathrm{n}$ hydrochloric acid at room temperature. Spontaneous gelation of the mixture occurs immediately after the complete addition of the acid. 
After drying at $130^{\circ} \mathrm{C}$, the sorption characteristics of the obtained solids were measured in an absorber column with butyric acetate, benzene, toluene and xylene as model solvents (column length: $300 \mathrm{~mm}$, diameter: $20 \mathrm{~mm}$ ). The flow rate of the air stream was $21 / \mathrm{min}$. The air speed calculated for the empty column was $10.6 \mathrm{~cm} / \mathrm{s}$. The volume of the composite in the column was $45.2 \mathrm{~cm}^{3}$, which gives an effective air speed of $20.4 \mathrm{~cm} / \mathrm{s}$. The concentration of the solvent in the air stream acting on the sensors leads to a change in the potential, which is detected. The surface areas of the solids were determined by BET analysis of nitrogen adsorption data, collected at $77 \mathrm{~K}$, measured with an ASAP 2400 from Micromeritics.

\section{RESULTS}

Both inorganic oranic composite coatings and bulk materials were synthesized via the sol gel process, but different paths were used. Figure 1 shows the reaction scheme for the synthesis of bulk materials and coatings.

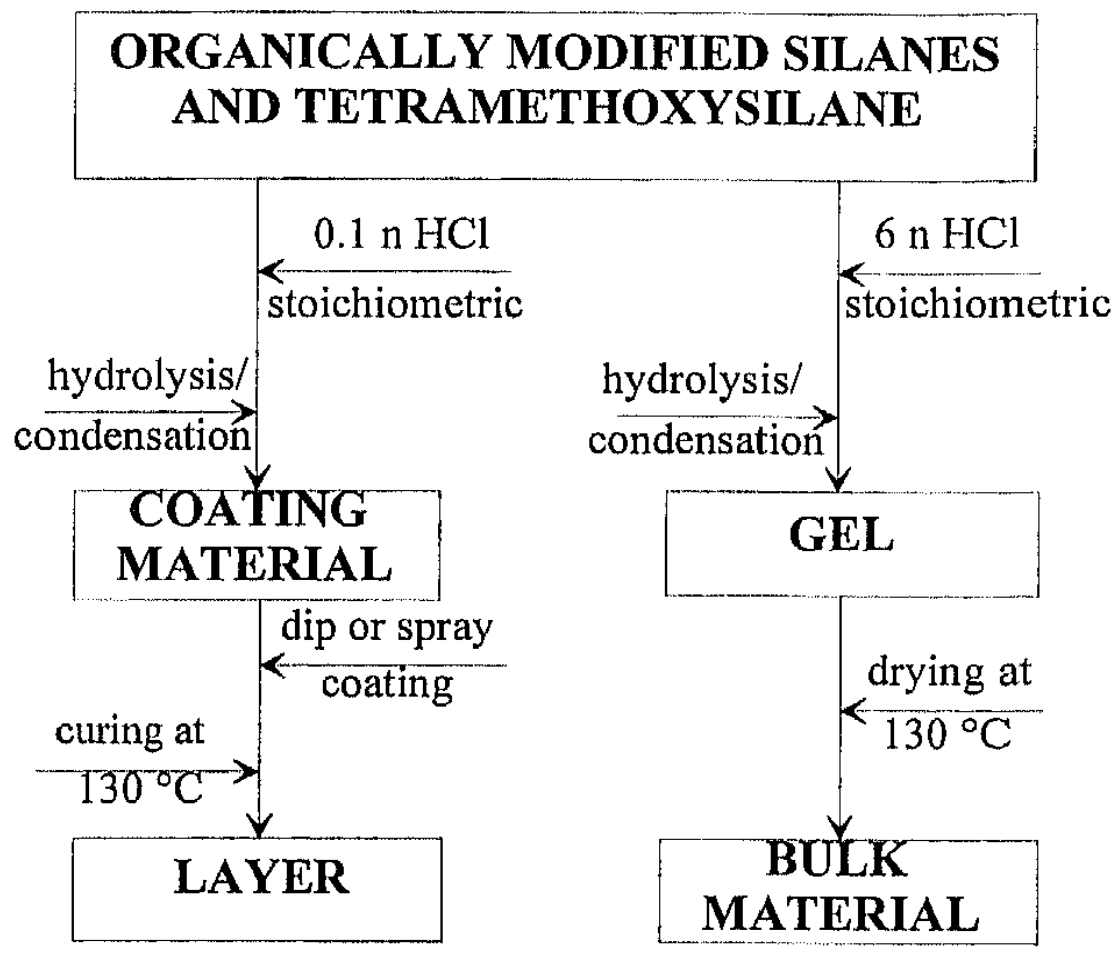

Figure 1: reaction scheme for the synthesis of inorganic organic composite coatings and bulk materials for absorption.

\section{Inorganic organic coating materials}

For the determination of the absorption characteristics, the takeup of the layers in a saturated solvent atmosphere was measured as a function of time. The butyric acetate takeup of the synthesized materials depends on the composition of the composites and is shown in figure 2. In particular, the content of different organic groups like methyl, propyl, phenyl or vinyl groups plays an important role on the capacity of such materials.

The high relative takeup observed with butyric acetate is remarkable since the coatings are non-porous and the specific surface area is correspondent to the film surface area. It can be 


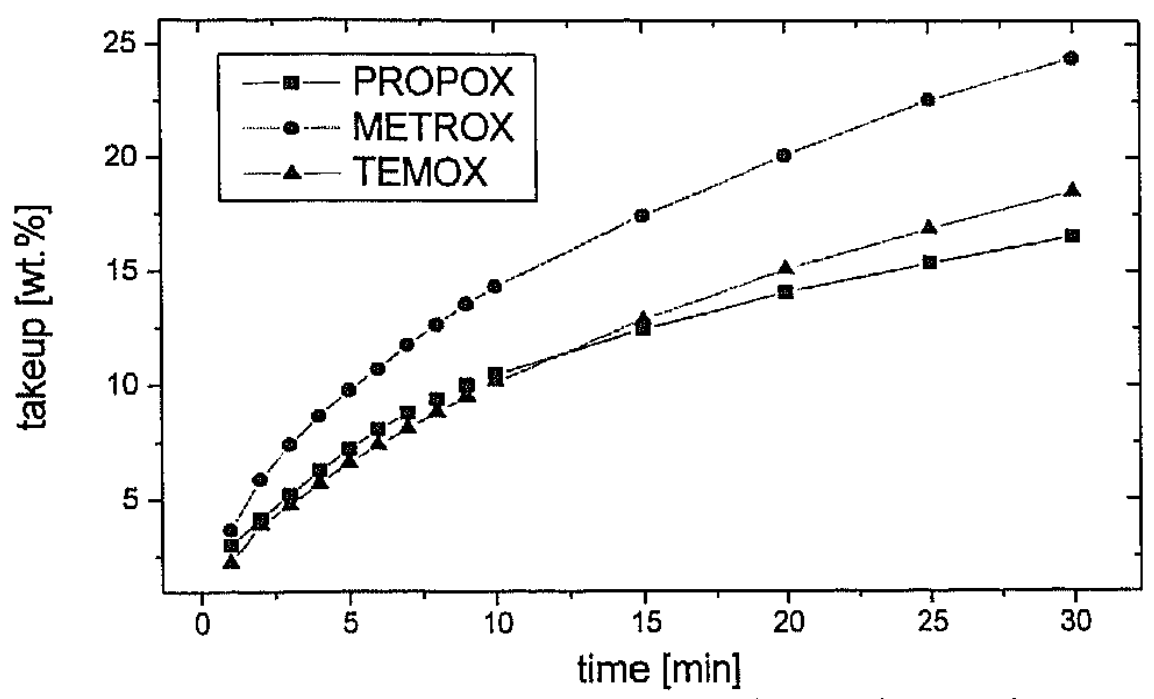

Figure 2: normalized takeup of butyric acetate of different inorganic organic composite layers (coating thickness: $5 \mu \mathrm{m}$ ), PROPOX: propyltrimethoxysilane; METROX: 25 mole \% methyltrimethoxysilane, 75 mole \% diphenyldihydroxysilane; TEMOX: 66 mole \% tetraethylorthosilicate, 17 mole \% diphenyldihydroxysilane, 17 mole-\% vinyltriethoxysilane

shown that the kinetics of solvent takeup are controlled by a diffusion limited process. The takeup can be described by equation (1) which can be derived from Fick's laws for diffusion [3].

$$
\frac{M_{t}}{M_{\infty}}=\frac{4}{b} \cdot\left(\frac{D}{\pi}\right)^{n}
$$

$M_{t}$ is the relative weight gain at the time $t, M_{\infty}$ is the relative weight gain in the equilibrium state, $D$ is the coefficient of diffusion, $b$ is the thickness of the layer and $n$ is a constant. For Fickian diffusion $\mathrm{n}$ is equal to 0.5 and the diffusion coefficient is independent of time. The plot of the takeup of butyric acetate versus $\sqrt{t}_{\mathrm{t}}$ shows that each of the coatings show a linear dependence which proves that the solvent takeup is a diffusion contolled process and takes place by absorption and not by adsorption (figure 3 ).

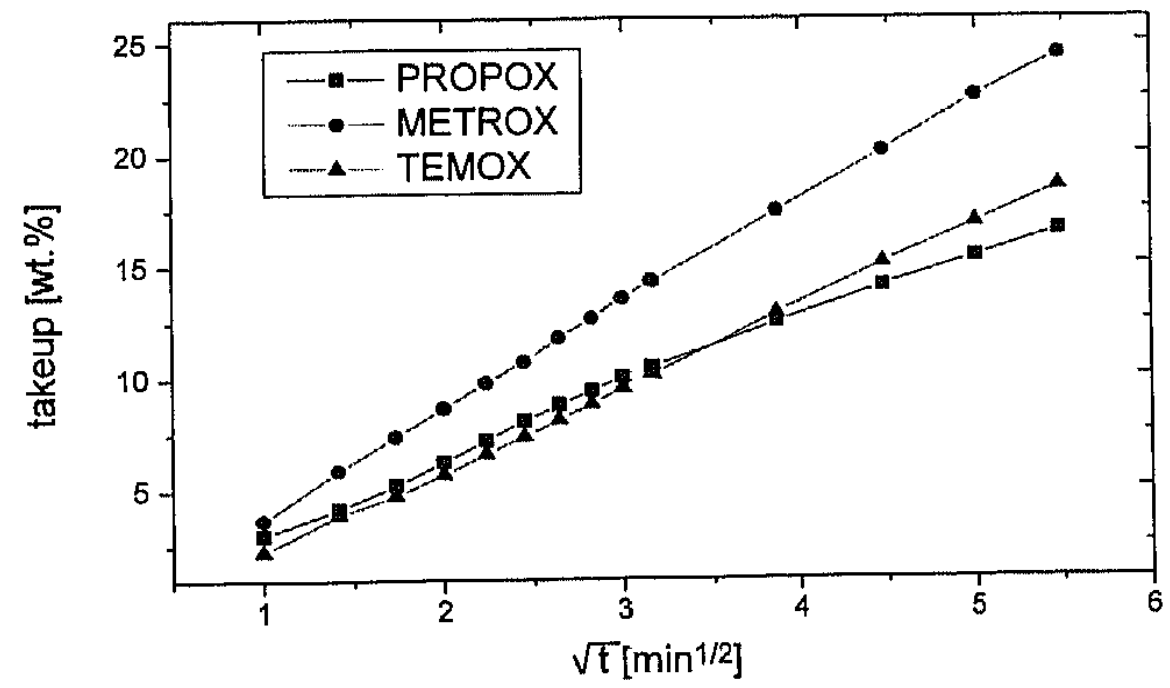

Figure 3: development of the takeup of butyric acetate of different coating systems as a function of $\sqrt{t}_{\mathrm{t}}$ 
In contrast to highly porous adsorbers such as activated carbon, where in many cases the complete desorption takes very long times ( $>1$ day) at elevated temperatures (in the range of 130 to $150^{\circ} \mathrm{C}$ ), the absorbed solvents can be desorbed completely from composite layers within a few minutes. Therefore the regenerative capacity of these coatings, which is of interest for practical application, is equal to the maximum takeup of a solvent after a given time of absorption. Figure 4 shows the absorption and desorption behaviour of butyric acetate on a composite layer.

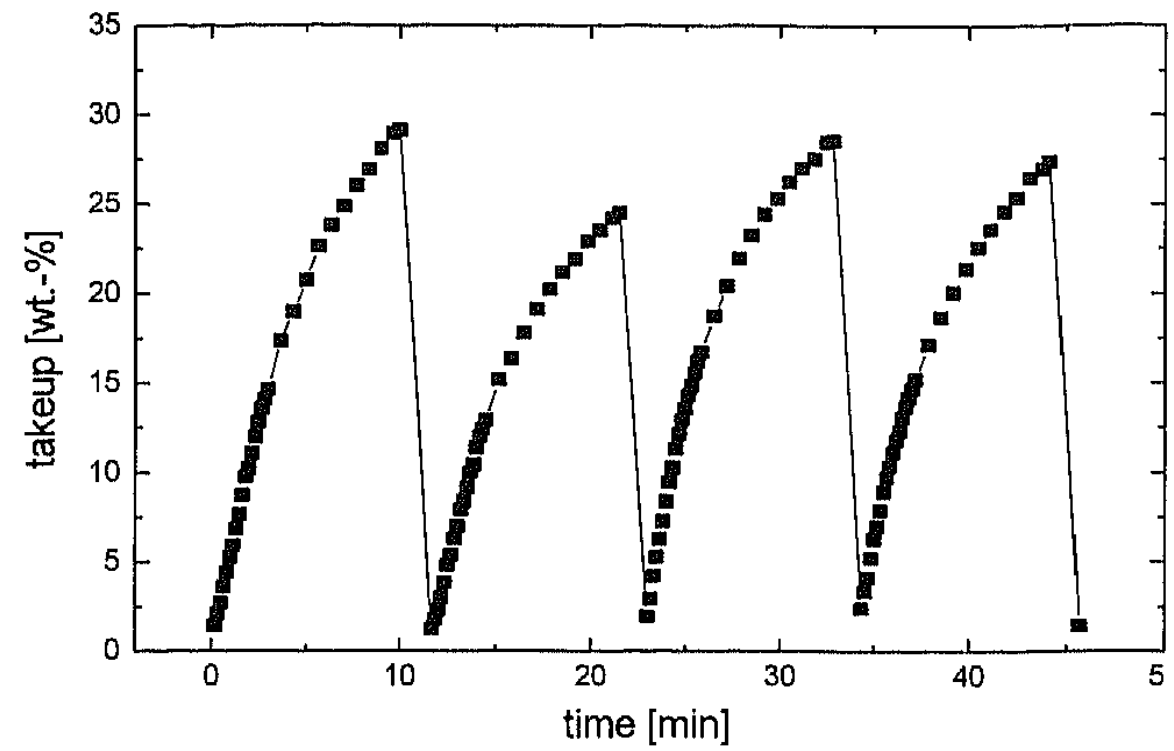

Figure 4: absorption and desorption behaviour of butyric acetate on a composite layer, layer-thickness: $1 \mu \mathrm{m}$, absorption at room temperature, desorption at $130^{\circ} \mathrm{C}$

The desorption time is a very important economic aspect. As can be seen in figure 4 the desorption time of composite layers is very short. By means of comparison, activated carbon has been investigated, which can adsorb up to $50 \%$ of solvent, needs more than $30 \mathrm{~h}$ for a complete desorption at the same temperature. Figure 5 shows as an example the desorption behaviour of activated carbon loaded with toluene at $130{ }^{\circ} \mathrm{C}$ compared to that of an inorganic organic composite layer.

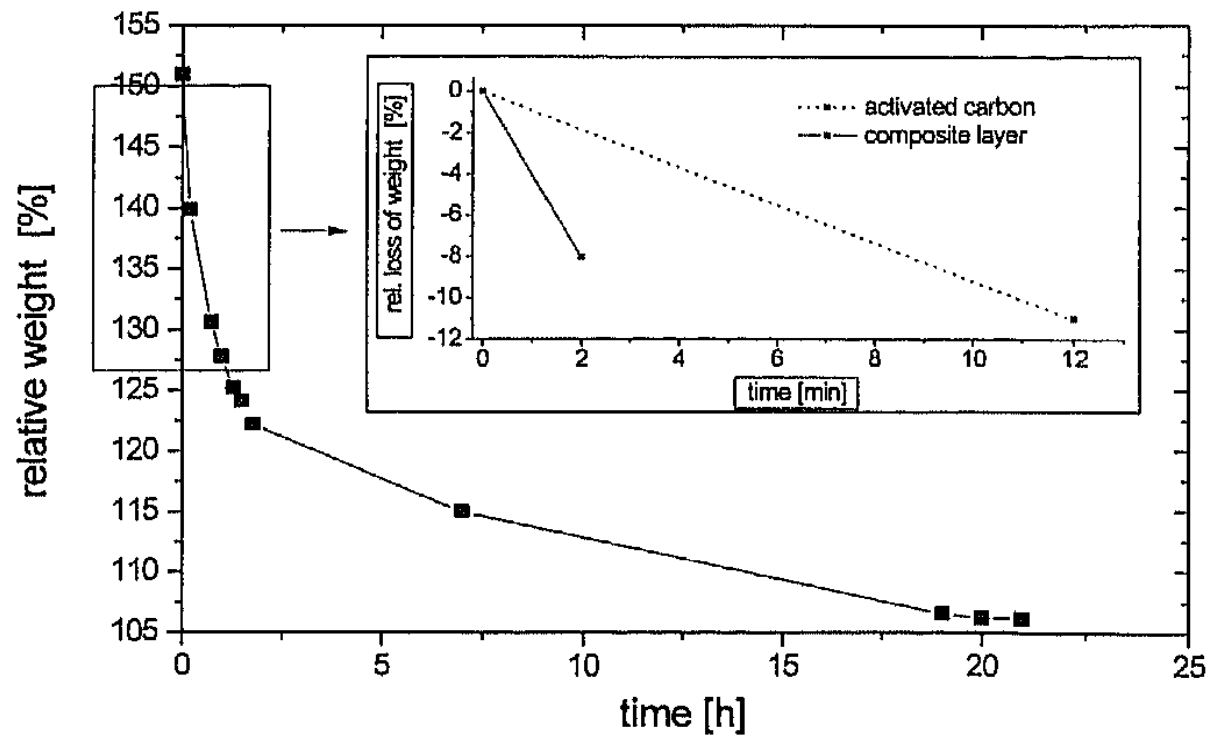

Figure 5: comparison of the desorption behaviour of activated carbon and a composite layer 
Since the solubility of a given solvent depends on the composition of the absorber material, it is possible to influence the selectivity of the composites for different solvents by tailoring the composition. The coating materials synthesized can be applied to different surfaces by common coating techniques, e. g. dip coating or spray coating, and can be cured thermally. To gain large capacities in combination with fast kinetics it is necessary to limit the thickness of the layers and to coat large surface areas, e. g. porous materials or structures similiar to an heat exchanger.

\section{Porous inorganic organic composite materials}

The aim of the investigations was the development of solid absorber materials with high regenerative capacities for organic solvents in comparison to activated carbon. The investigation of absorbing composite coatings has shown that a maximum solvent takeup of $30 \mathrm{wt} . \%$ can be achieved within 30 minutes. Based on these results, inorganic organic composite bulk materials were developed, which can be used as absorption materials for organic solvents. Solid phases derived from methyl substituted silanes and tetramethylorthosilane, which have BETsurfaces of more than $700 \mathrm{~m}^{2} / \mathrm{g}$, show the best results in solvent takeup. As with the coating materials, butyric acetate, benzene, toluene and xylene were chosen as solvents for the investigations. A maximum takeup of solvent of nearly $38 \mathrm{wt} \%$ within $2-3$ hours can be observed depending on the composition of the materials. For the determination of the absorber characteristics, the materials were placed in a column, which is flooded with an air stream loaded with the appropriate organic solvent. The solvents are detected at the entrance and the top of the column by sensors as shown in figure 6 .

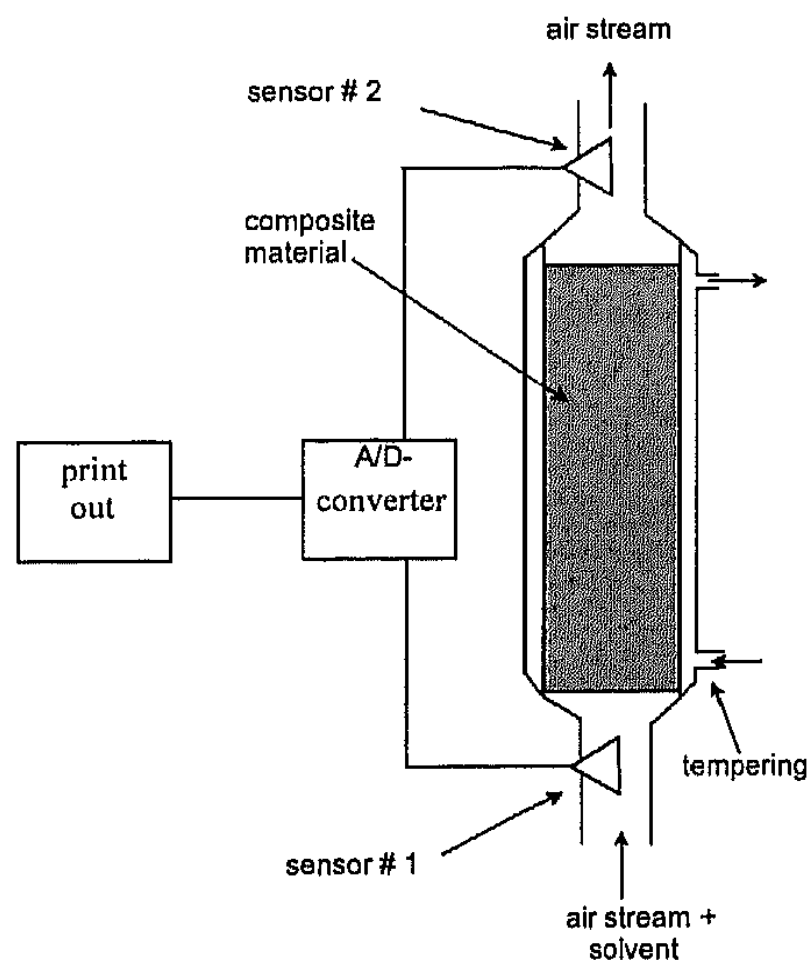

Figure 6: scheme of the apparatus for absorption measurements on bulk materials

As can be seen in figure 7, the solvent is completely held up by absorption for over 2.5 hours. The capacity at the point of breakthrough is $30 \%$. 


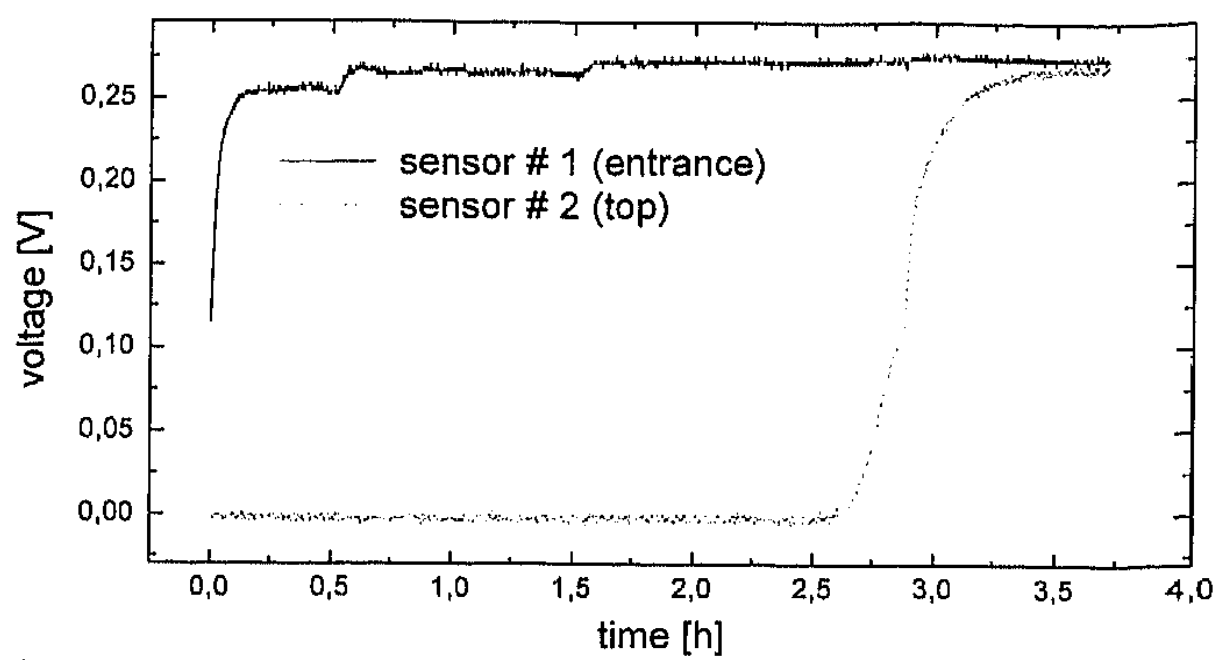

Figure 7: development of the sensor signals at the entrance and the top of the column as function of time, flow rate: $2 \mathrm{l} / \mathrm{min}$, vapour pressure of the solvent (butyric acetate): $52 \mathrm{mbar}$

The desorption takes place at a temperature of $130{ }^{\circ} \mathrm{C}$, whereby $90 \%$ of the absorbed solvent can be removed in the first hour. The complete desorption takes $4-5$ hours. In comparison, activated carbon with a specific surface of $1300 \mathrm{~m}^{2} / \mathrm{g}$, loaded with $50 \%$ of solvent, requires more than 30 hours for a complete desorption at the same temperature. Absorption experiments with wet air show that the water takeup of the bulk material developed is in the same order of magnitude as the takeup of organic solvents. By comparison, composite coatings show a water takeup of only $2-3 \mathrm{wt} . \%$. The large specific surface area of the bulk materials, which is caused by the high porosity, could be the reason for this behaviour. If the surface of the material is covered with water due to adsorption and capillary condensation in the pores only a small part of the surface is able to absorb non polar organic solvents. This leads to very long diffusion paths and to slow absorption kinetics, which results in a rapid break through of solvents in the column. To avoid this the specific surface area, the pore structure and the polarity of the surface of the materials have to be tailored.

\section{CONCLUSION}

The investigations have shown that both inorganic organic composite coatings and bulk materials are able to absorb organic solvents from the gas phase. Compared to classic adsorbers the maximum capacities of $30 \mathrm{wt} . \%$ with coatings and $38 \mathrm{wt} . \%$ with bulk materials are not as high as that of e g. activated carbon but the necessary desorption time is much shorter than that of activated carbon.

\section{REFERENCES}

1. H. Schmidt, A. Kaiser, M. Seiferling; presented at the Enviceram '91, 2. Int. Symposium, Saarbrücken, Germany, 1991 (unpublished).

2. V. Gerhard, U. Dreier, H. Schirra, H. Schmidt; presented at the workshop "Teschnologie und Anwendung von Sol-Gel-Schichten, Frankfurt a. M., Germany, 1996 (unpublished).

3. Gad Marom; Polymer Permeability, Belfast University Press, Belfast, 341 (1985). 